\title{
Supplementary Information for: Modulation of Low-Frequency Acoustic Vibrations in Semiconductor Nanocrystals through Choice of Surface Ligand
}

A. Jolene Mork ${ }^{1}$,Elizabeth M.Y. Lee ${ }^{2}$, Nabeel S. Dahod ${ }^{2}$, Adam P. Willard ${ }^{1}$, William

\author{
A. Tisdale ${ }^{2 *}$
}

\section{Nanocrystal Characterization:}

QD size was measured by transmission electron microscopy (TEM) and confirmed with small-angle $\mathrm{x}$-ray scattering (SAXS). A sizing curve was constructed based on TEM measurements of 40 samples (Figure S1). Sizes measured using SAXS were generally found to agree with TEM measurements to within $\leq 0.2 \mathrm{~nm}$. Example TEM images and SAXS patterns are shown in Figure S2.

Transmission electron microscopy (TEM) was performed on a JEOL 2011 instrument operating at $200 \mathrm{kV}$. Samples were prepared by drop casting QD suspensions in hexanes onto copper TEM grids coated with an amorphous carbon support film. Nanocrystal sizes were extracted via image-analysis techniques previously reported [1]. Solution-phase small-angle X-ray scattering (SAXS) was measured on a Rigaku Smartlab with a $\mathrm{Cu} \mathrm{K} \alpha$ source operating at $45 \mathrm{kV}$ and $200 \mathrm{~mA}$ in transmission geometry with convergent beam optics and a $2 \mathrm{~mm}$ length limiting slit inserted. Samples were prepared by filling glass capillaries with suspensions of QDs in toluene at concentrations greater than $50 \mathrm{mg} / \mathrm{mL}$. The experimental SAXS data were fit using Rigaku NANO-Solver software to model the form factor of spherical CdSe nanocrystals in a matrix of toluene. The background scattering from a pure toluene capillary was subtracted from the experimental data. The software also accounted for instrumental smearing. 

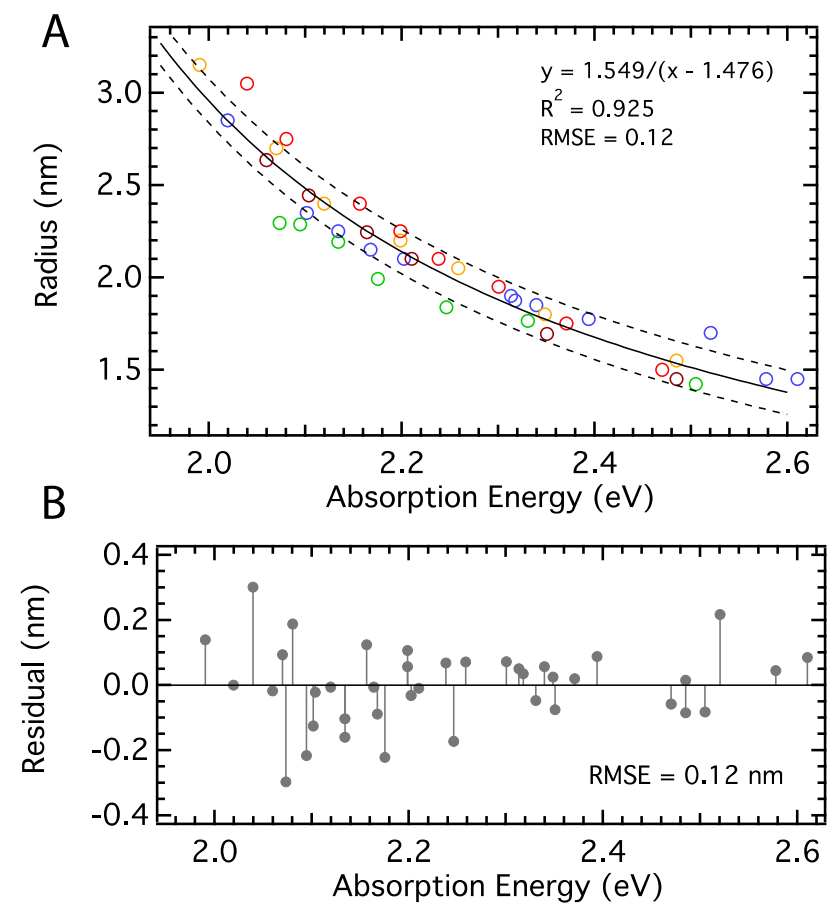

FIG. S1. (A) Plot of QD radii measured by TEM against the first absorption peak energy with a fit line corresponding to the equation $\mathrm{R}=1.549 /(\mathrm{AbsPeak}-1.476)$ as a solid black line. Dotted lines represent the best fit \pm RMS error. Different colors represent different syntheses where aliquots were taken over time during the QD synthesis. (B) Residuals of the fit, with root-mean-square error $0.12 \mathrm{~nm}$. This sizing curve and error were used throughout the manuscript for QD sizes. 

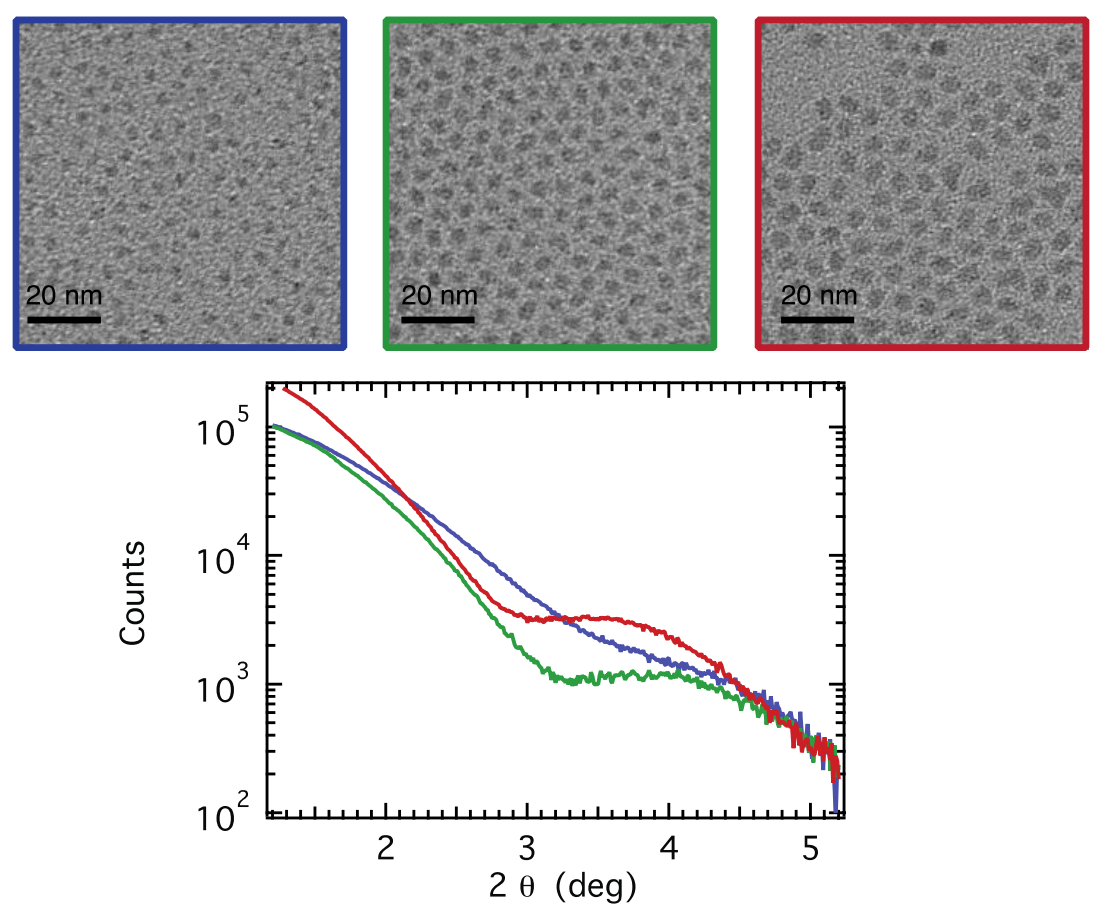

FIG. S2. Top: TEM images of three different sizes of QDs, ranging from small $(3.1 \mathrm{~nm})$ to large $(4.5 \mathrm{~nm})$. Bottom: SAXS patterns for the three different sizes of QDs with the same line colors as box colors for TEM images.

\section{Alkanethiol Ligand Exchange}

QDs were ligand exchanged with variable length alkanethiols (hexanethiol, octanethiol, decanethiol, dodecanethiol, tetradecanethiol, octadecanethiol) purchased from Aldrich and used as received. For the ligand exchange reaction, 20-50 nmol of freshly purified CdSe QDs were dissolved in $1 \mathrm{~mL}$ of chloroform. To this solution were added $1 \mathrm{mmol}$ of the desired alkanethiol and $0.1 \mathrm{mmol}$ of triethylamine to serve as a non-interacting base to help generate the alkyl thiolate. Ligand exchange solutions were stirred overnight $(>12 \mathrm{hrs})$ and the QDs were subsequently crashed out with a combination of methanol and butanol. Purified QD samples were resuspended into hexanes and drop cast onto clean glass coverslips and allowed to dry before collecting Raman spectra.

To confirm the success of ligand exchange, we measured the ${ }^{31} \mathrm{P}$ and ${ }^{1} \mathrm{H}$ NMR spectra for dodecanethiol-exchanged QDs and compared them to the spectra acquired for control (non-ligand exchanged) QDs from the same batch synthesis. Both were purified by crashing out 4 times, then dried under vacuum before NMR analysis. The ligand- 
exchanged QDs and the control QDs were diluted to the same concentration into

chloroform-d, and the ${ }^{1} \mathrm{H}$ and ${ }^{31} \mathrm{P}$ acquisition parameters were constant for all spectra. As can be seen in Figure S3, the phosphorus ligand bound to the surface of the control QDs largely disappears in the ligand exchanged QDs. Based on absolute intensity integration of these peaks, the ligand exchange was effective at removing $\sim 99 \%$ of the native ligands from the sample surface.

\section{B. Benzylmercaptan Ligand Exchange}

QDs were ligand exchanged with various para-substituted benzylmercaptans, with general structure shown in the inset of Figure S4b. The sulfur atom that binds the QD is separated from the phenyl ring by a methylene spacer to minimize electronic interactions between the para-substituent and the thiol binding group. Ligand exchange with benzylmercaptans proceeded exactly as with alkanethiols except during the purification. QDs substituted with benzylmercaptans with the para-substituent $\mathrm{X}=\mathrm{H}, \mathrm{F}, \mathrm{Cl}, \mathrm{Br}, \mathrm{CF}_{3}$ were crashed out for purification by adding hexanes, and were resuspended in acetone. QDs substituted with benzylmercaptans with $\mathrm{X}=\mathrm{Me}$, $\mathrm{tBu}$ were crashed by adding methanol and butanol and were resuspended in hexanes. After purification and resuspension, these samples were drop cast onto clean glass coverslips and allowed to dry before collecting Raman spectra.

\section{Ligand Exchange does not Alter QD Size}

To verify that observed changes in the QD Raman spectra were not due to changes in QD size, absorption spectra for all QD samples before and after ligand exchange were collected and compared. As seen in Figure S4, the absorption onset remains constant after ligand exchange. Quenching of the second absorption feature is seen, which has been suggested to indicate a mixture of ligands at the surface. 

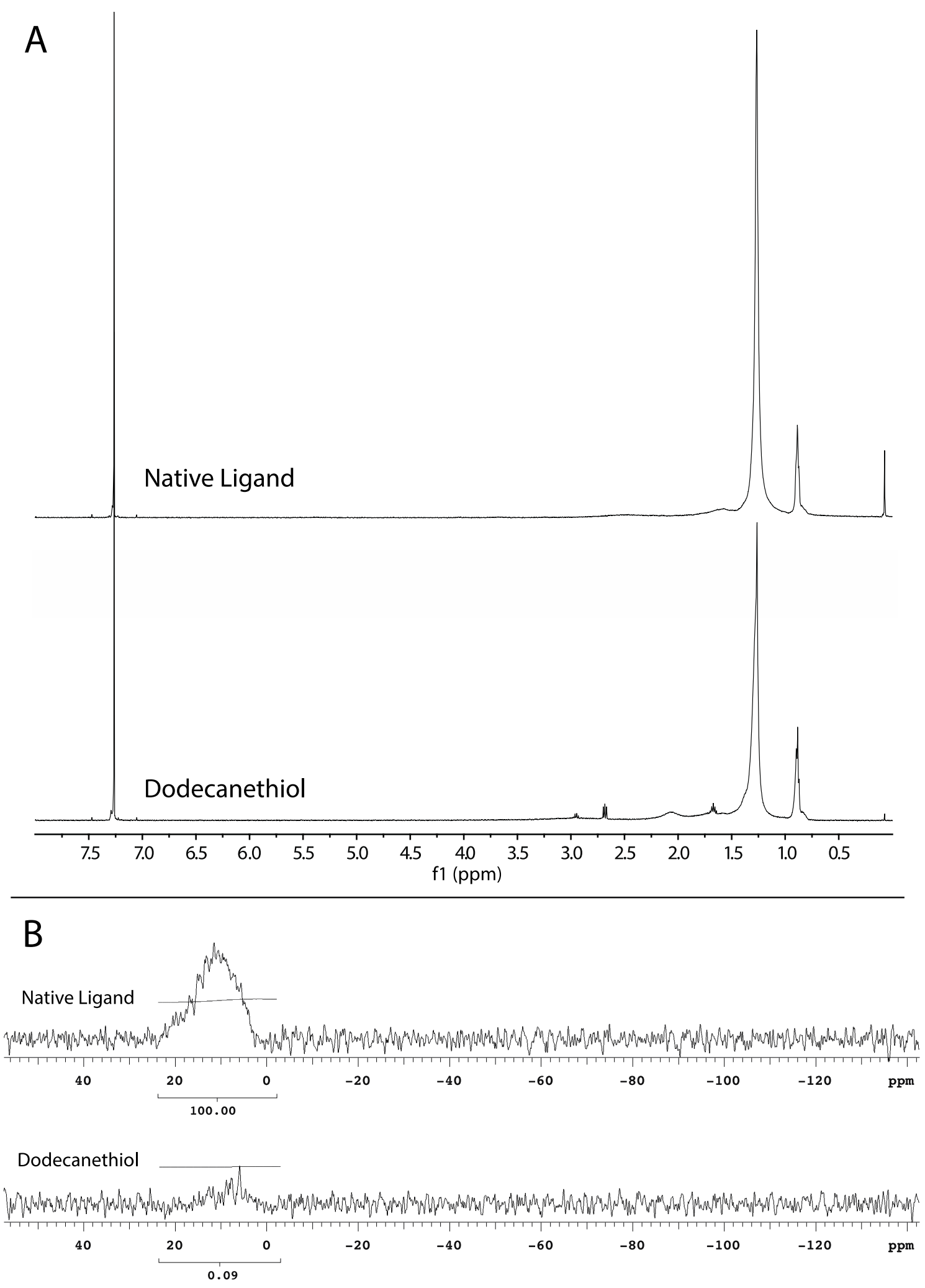

FIG. S3. (A) ${ }^{1}$ H NMR spectra of QDs with native phosphonic acid ligands (top) and after 
ligand exchange with dodecanethiol (bottom). Proton NMR spectra do not allow sufficient differentiation between alkanethiol and native ligand peaks to permit quantification of ligand exchange. (B) ${ }^{31}$ P NMR spectra of QDs with native ligands (top) and QDs after ligand exchange(bottom). The cores with native ligands show a characteristic broad phosphoric acid peak between 0-20 ppm, and this peak has largely disappeared in the ligand exchanged sample. The phosphorous peaks have been integrated on the same absolute integral scale, and comparison shows that the ligand exchange procedure was $99 \%$ effective in removing the native ligands from the QD surface. The concentration of QDs (determined by UV-Vis spectroscopy) and the acquisition parameters were the same for both samples.
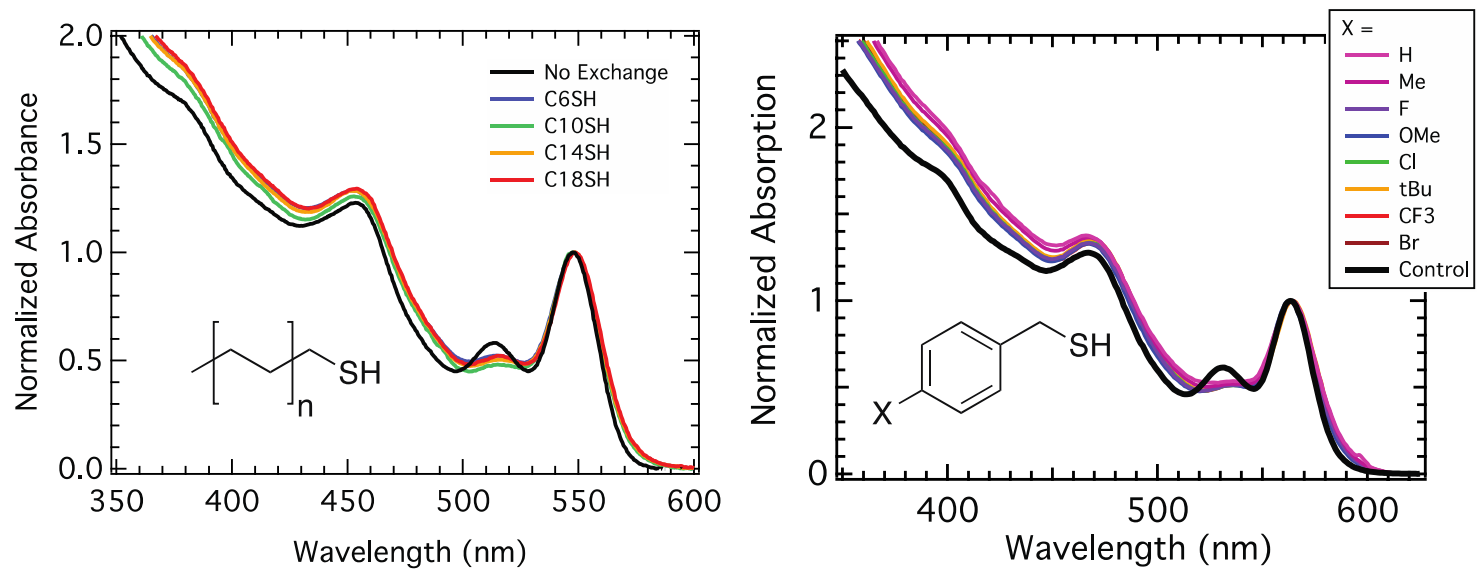

FIG. S4. QDs after ligand exchange with alkanethiols (left) and benzylmercaptans (right) of different sizes show no change in the absorption onset, which indicates the QD size remains constant upon ligand exchange, and the ligand exchange does not affect the QD Raman spectrum through changing QD size. 


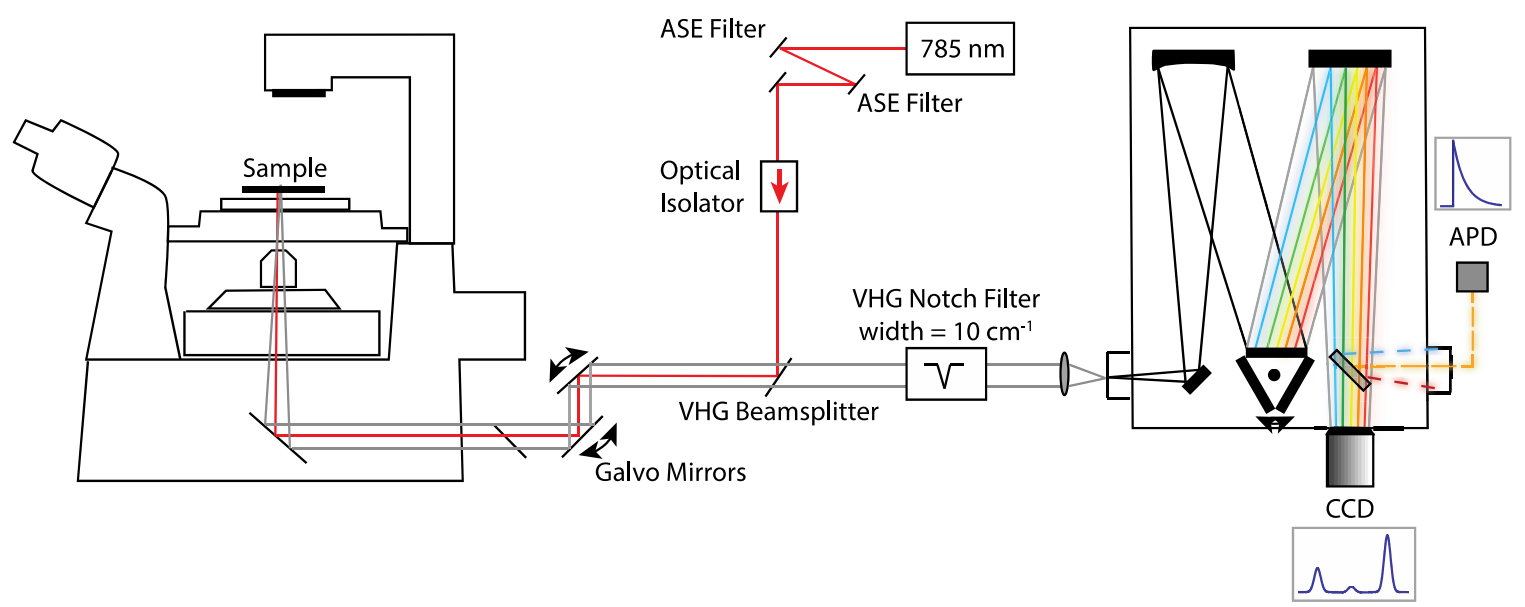

FIG S5. Schematic of low-frequency Raman microscopy setup used in this work.

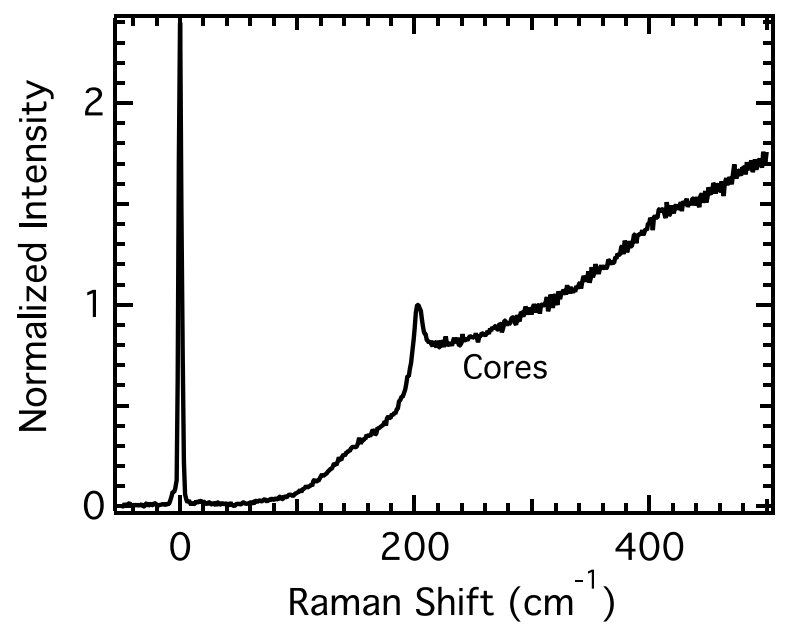

FIG S6. Resonant Raman experiment $(532 \mathrm{~nm})$ of the same nanocrystals used in the main text, revealing the characteristic LO phonon mode near $210 \mathrm{~cm}^{-1}$. The rising background is QD photoluminescence due to above-bandgap excitation.

[1] M. C. Weidman, M. E. Beck, R. S. Hoffman, F. Prins, and W. A. Tisdale, ACS Nano 8, 6363 (2014) 